\title{
THE DEVELOPMENT OF AN INFORMATION SHARING LINKAGE SCALE FOR SERVICE SECTOR
}

\author{
Siti Aisyah Ya'kob* \\ Universiti Malaysia Sarawak \\ Jamal Abdul Nassir Shaari \\ Universiti Sains Islam Malaysia \\ Nor Afiza Abu Bakar \\ Universiti Malaysia Sarawak
}

\begin{abstract}
This study was done to evaluate the validity of an information sharing linkage scale which will be used for small and medium enterprises (SMEs) in service sector. Content validity is evaluated by six experts in the field and quantified as content validity index (CVI). Content validity assessment is inclusive of four rating criteria which are relevance, clarity, simplicity and ambiguity. All items that fulfil the criteria of content validity index which is at least 0.75 are usable for research instrument. The final version of linkages was improvised in 35 items with 3 factors for information sharing linkage i.e. customer linkage, supplier linkage, and internal linkage; 2 factors for firm performance i.e. operational performance and financial performance.
\end{abstract}

Keywords: Content Validity Index; Information Sharing Linkage; Operational Performance; Financial Performance; Service Sector.

\section{INTRODUCTION}

Linkage is a business practice that enables the organization to complete the business activities and excel in business transactions (Samiee, Yip, \& Luk, 2004). Looking at supply chain linkages from information gaining perspective, three types of linkages could be identified: internal linkage (Lee, Kwon, \& Severance, 2007), supplier linkage (Ibrahim \& Ogunyemi, 2012; Lee et al., 2007) and customer linkage (Ibrahim \& Ogunyemi, 2012; Lee et al., 2007). These three linkages are obtained from studies on manufacturing sector. Unlike the previous studies, this paper is focusing on information linkage on service sector. The different nature of service-based businesses which offers intangible value to customers compared to the manufacturing-based which is more tangible requires an investigation on information linkages in service sector which is logically to be different from the manufacturing side. Hence, this results in the need to modify the previous measurement scale; which is in this context was adapted from Ibrahim and Ogunyemi (2012) and Lee et al. (2007) to fit with the focus of this study. This is in accord to Rungtusanatham (1998) that content validity needs to be assessed as the prior empirical studies are not sufficient to conclude the content validity (Rungtusanatham, 1998).

\footnotetext{
- Corresponding author: Siti Aisyah Ya'kob, Faculty of Economics and Business, Universiti Malaysia Sarawak, 94300 Kota Samarahan, Sarawak, Malaysia. Tel: +6082-581000 ext.4383. Email: ysaisyah@unimas.my
} 
Polit and Beck (2006) further assert that content validity is a requirement in scale development. Considering the essential of developing the empirical measurements in initial stage (Polit \& Beck, 2006; Rungtusanatham, 1998; Wynd, Schmidt, \& Schaefer, 2003) and researchers' concerns about the instruments (Yaghmaie, 2003), this paper sets for two objectives. First is to contribute to further highlight the importance of content validity; and second is to report the assessment of the content validity of the researchers' information sharing linkage scale developed specifically for service sector in small medium enterprises in Malaysia. The assessment is done by applying the quantitative method. The content validity assessment is inclusive of four rating criteria which are relevance, clarity, simplicity and ambiguity of the items.

\section{LITERATURE REVIEW}

\subsection{The Concept of Content Validity}

Content validity has been known with several terms such as intrinsic validity, representative validity, content-related validity, logical validity and relevance validity (Yaghmaie, 2003). Content validity is not a new measurement procedure and has been theoretically discussed years ago. In 1970's, researchers have discussed on content validity as a perseverance to examine "the representative of the small sample of behaviour observed in the measurement procedure" (Guion, 1977). Yaghmaie (2003) has empirically proven the assessment of content validity and improvised the concept to make it in line with the functions of testing the measurements.

From quantitative judgement-based definition, content validity can be denoted as "the degree to which elements of an assessment instrument are relevant to and representative of the targeted construct for a particular assessment purpose" (Haynes, Richard, \& Kubany, 1995). According to Sireci (1998) the content validity also refers to the score-based inferences of the content representativeness, where else from another perspective, Rungtusanatham (1998) presumes content validity as "the extent to which a measurement instrument captures the different facets of a construct". As the judgement and inference are based on score or rating, the level of relevant items in an instrument assessment is subjective according to the experts' evaluation (Yaghmaie, 2003). Based on the explanations given, the authors contextualize content validity as concerns on the content relevancy and the representative of the measurement instrument with subjective judgement from the experts of the area.

\subsection{The Importance of Content Validity}

As mentioned earlier, in past studies, content validity was applied in many areas which includes psychology (Guion, 1977; Haynes et al., 1995), education (Elanna \& Popham, 1983), business and management (Lawshe, 1975; Lloyd, 2011; Rungtusanatham, 1998) as well as medical and health (Wynd et al., 2003; Yaghmaie, 2003). Notwithstanding the availability of these previous empirical studies, we agree with Rungtusanatham (1998) that they are not sufficient to highlight the importance of having content validity assessment for researchers. In fact, assessment on content validity has not obtained high consideration in many empirical studies which is evident with little literatures report on results of content validity (Yaghmaie, 2003). Therefore, from available literatures, the authors highlight below some of the obvious reasons of why it is important to have content validity, which is further strengthened the raison d'etre of this paper: 
a. In developing the measurement instrument, assessing content validity is important during the initial stage for linking the concepts with measureable indicators (Wynd et al., 2003) and validating the content of the new instruments (Wynd et al., 2003; Yaghmaie, 2003)

b. Content representativeness can be identified in content validation by measuring the sampling of the content domain of items in an instrument (Sireci, 1998; Yaghmaie, 2003)

c. Content validity enables the researcher to obtain accurate information regarding the quality of the measurements (Elanna \& Popham, 1983; Polit \& Beck, 2006)

d. The relevance of measurements to the domain of content can be examined (Wynd et al., 2003; Yaghmaie, 2003)

\subsection{Content Validity Procedure}

A study on content validity is done based on the previous theoretical and empirical studies. Content validity index or simply called as CVI was first developed by Waltz and Bausell in 1981. The application of content validity index (Lawshe, 1975; Polit \& Beck, 2006; Wynd et al., 2003; Yaghmaie, 2003) refers to the ratings of item relevance to the particular study as being measured by content experts (Polit \& Beck, 2006). Polit and Beck (2006) highlight on two calculation methods known as the content validity index for scales (S-CVI) and content validity index for items (I-CVI).

\subsubsection{Four-point Rating}

Yaghmaie (2003) applied a four-measured criteria of content validity that involves relevance, clarity, simplicity and ambiguity of the content which is named as content validity index (CVI). The CVI is measured by a four-point rating (Polit \& Beck, 2006; Yaghmaie, 2003). Polit and Beck (2006) and Wynd et al. (2003) approaches in four-point rating is by dichotomizing the scale into two categories. On one hand, rating 1 and 2 represent invalid content or not relevant, while on the other hand, rating 3 and 4 represent valid content or relevant. The relevance of content validity is related to the level of specificity of the target construct and the degree to which the main focus is on the obtained measure (Haynes et al., 1995). It is possible for researchers to refer to invalid or irrelevant content. Data obtained from invalid instrument can mislead some facets of constructs and reflects the items outside the construct domain (Haynes et al., 1995). The criteria of CVI and four-point rating are illustrated in Table 1. In CVI procedure, items with acceptable level of relevance will remain, while those items with unacceptable level of relevance or invalid need to be revised or eliminated (Polit \& Beck, 2006; Wynd et al., 2003; Yaghmaie, 2003).

Table 1: Content Validity Index (CVI) Criteria and Four-Point Rating

\begin{tabular}{ccccc}
\hline \hline Criteria & \multicolumn{3}{c}{ Four-point Rating } \\
\hline Relevance & 1 & 2 & 3 & 4 \\
& Not relevant & Item needs some revision & Relevant but needs minor revision & Very relevant \\
Clarity & 1 & 2 & 3 & 4 \\
& Not clear & Item needs some revision & Clear but needs minor revision & Very clear \\
Simplicity & 1 & 2 & 3 & 4 \\
& Not simple & Item needs some revision & Simple but needs minor revision & Very simple \\
Ambiguity & 1 & 2 & 3 & 4 \\
& Doubtful & Item needs some revision & No doubt but needs minor revision & Meaning is clear \\
\hline \hline
\end{tabular}

Source: Adopted from Yaghmaie, 2003. 


\subsubsection{Evaluation of experts}

Content validity requires evaluation of expert in subject-matter (Lawshe, 1975; Rungtusanatham, 1998). Hence, a thorough process of selecting the expert raters is needed, commonly involving the academicians and professionals (Lawshe, 1975). In consideration of the greater understanding and insight into the subject-matter (Lawshe, 1975), the experts are expected to be competent to review and assess the items of the dimension independently (Wynd et al., 2003). To validate the measurements for factors, Lloyd (2011) invites the professionals and practitioners with interest and direct involvement in the subject-matter as the expert raters. This means that, the expert in subject-matter is not only restricted to academicians, but could also involve relevant individuals who are able to review and assess items to the particular dimensions.

After expert raters are determined, they are invited to participate in the study. A copy of the measurements is given and the purpose of the study is justified (Yaghmaie, 2003). The expert raters are to rate the items with four-point rating to indicate the level of content validity and to identify the overall CVI (Polit \& Beck, 2006; Wynd et al., 2003; Yaghmaie, 2003). A total 100 percent agreement from all expert raters are not expected in consideration of some aspects such as the biased viewpoint of the experts (Polit \& Beck, 2006). When compiling the experts' evaluation, items which have acceptable level of relevance will be kept while revision for the items which have unacceptable level of relevance is done according to expert raters' evaluation and the elimination of those items is made if the items are considered not related to the dimension (Wynd et al., 2003; Yaghmaie, 2003).

\section{METHODOLOGY}

This study adopts a four-point content validity index (CVI) in conducting the content validity procedure. To generate a representative sample of items for information sharing linkage and business performance, the items have been adopted from previous literature review of Flynn, Huo, and Zhao (2010) and Lee et al. (2007). At this stage, the measurements for information sharing linkages (Lee et al., 2007) were validated with 20 items and the measurements for business performance were validated with 13 items (Flynn, et al., 2010). To test the content validity, eleven academicians from public universities and ten professionals from relevant institutions were invited to be the expert raters. They were given three-week duration to complete the content validity process. From the total of twenty-one expert raters, five academicians and one professional have successfully completed the validity procedure. The experts from academic field are chosen based on their understanding and knowledge on the subject-matter meanwhile the professionals are invited in consideration of their experience and involvement in the industry. An invitation letter explaining the purpose of conducting the study was sent and a copy of the measurement was attached together with the letter. CVI instrument applied in Yaghmaie (2003) has been adopted in this study. The experts need to rate each item based on four criteria which are relevance, clarity, simplicity and ambiguity on the four-point rating as shown in Table 1. The responses from academicians and professionals were then analyzed by adopting the methods named as 'content validity index' as discussed in earlier section.

\section{DATA ANALYSIS AND FINDINGS}

\subsection{Content Validity Index}

To validate the content validity, an analysis was conducted to assess the relevance, clarity simplicity and ambiguity of instrument measurements. The mean score of content validity is found by analyzing 
responses from all 6 expert raters. In total, the expert raters evaluated 33 items that consist of information sharing linkage i.e. customer linkage, supplier linkage, and internal linkage and business performance i.e. operational performance and financial performance.

The authors are in agreement with Polit and Beck, (2006) that in consideration of biased viewpoint of the experts we were not expecting results of 100 percent agreement. Hence, we follow Yaghmaie (2003) that items with CVI of at least 0.75 are kept while those with CVI less than 0.75 are removed. By removing those variables that have low CVI, the number of items for information sharing linkage decreased from 20 items to 16 items. In detail, customer linkage is made up of four items and both supplier linkage and internal linkage are made up of six items respectively. Additionally, the numbers of items for business performance were also reduced from 13 items to 10 items. To be precised, operational performance is made up of 3 items and financial performance is made up of 7 items. These are items that fulfil the criteria of content validity index which is at least 0.75 , which are considered usable for research instrument as presented in Table 2.

Yaghmaie (2003) suggests that, item should have CVI of at least 0.75 to be accepted and to remove items with CVI score below 0.75 . The seven items which were removed from the analysis are shown in Table 3.

\subsection{Improvisation of Research Instrument}

Next, the remaining 16 items of information sharing linkage and 10 items of business performance were modified according to the comments from the experts in relation with the service sector for small and medium enterprises. This improvisation is expected for the reason as discussed earlier that since the research instruments were adopted from a study on manufacturing sector, variances have been identified and improvisation of items are required due to the dissimilar nature of services and manufacturing-based businesses. As recommended by the experts, several items require revision due to double-barrel, use of jargons, unsuitability and ambiguity of the items. An improvised version of research instrument has been done following the content validity index and experts' recommendation. As a result, the improvised version of instrument consist of 9 items to measure both customer linkage and internal linkage, and 7 items to measure supplier linkage. There are 7 items for financial performance and 3 items to measure operational performance. The improvised version of research instrument is presented in Table 4.

\section{DISCUSSION AND CONCLUSION}

As the importance of conducting a comprehensive content validity of research instrument was highlighted in previous studies (Elanna \& Popham, 1983; Polit \& Beck, 2006; Rungtusanatham, 1998; Sireci, 1998; Yaghmaie 2003, Wynd et al., 2003), a thorough procedure of content validation has been done to obtain the relevance, clarity, simplicity and no-ambiguity of items used in research instrument. Supporting Yaghmaie (2003), the undertaking of content validity analysis for information sharing linkage could provide support for previous studies on importance of having content validity of an instrument. 


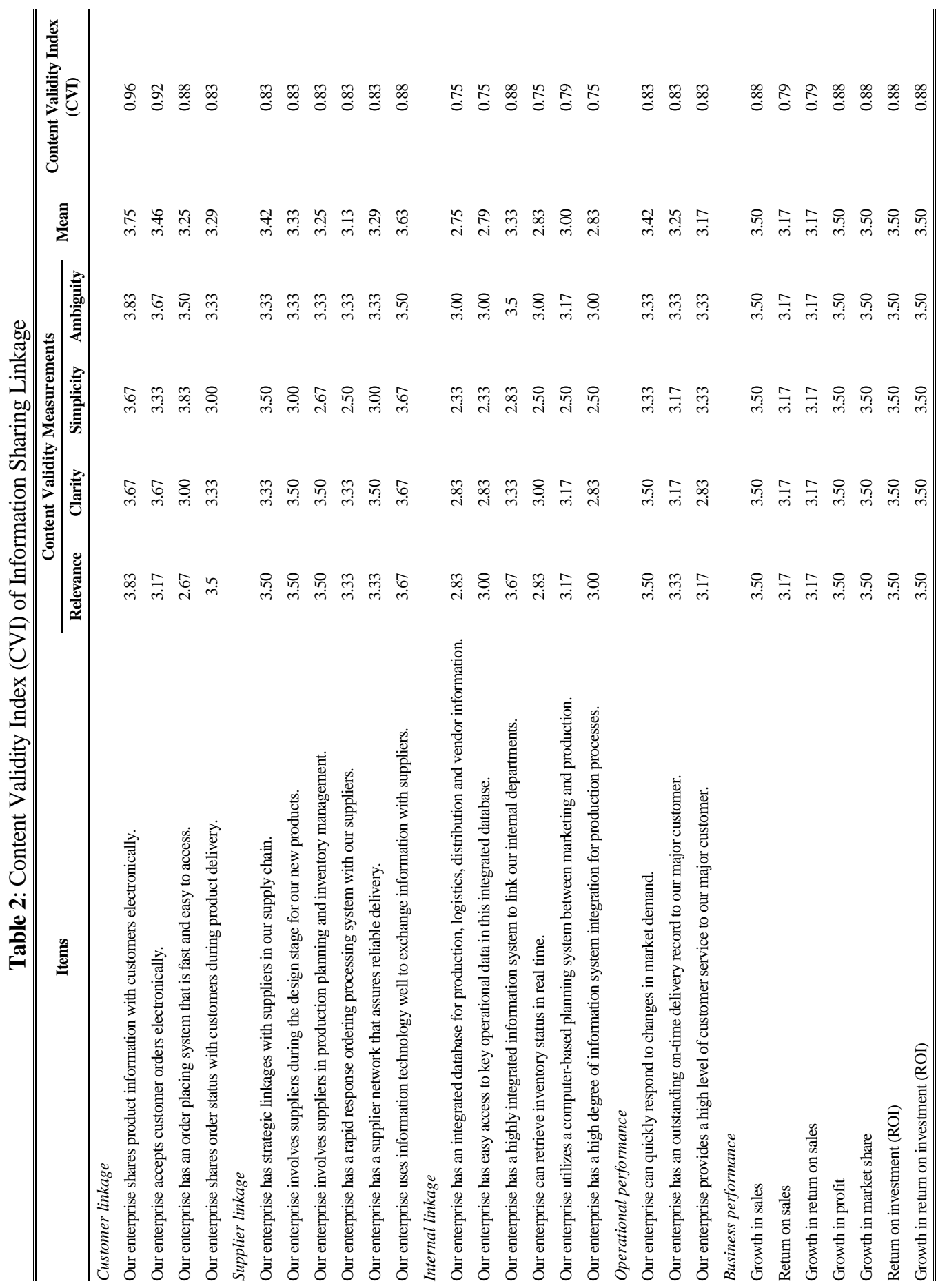


Table 3: Items Removed by Content Validity Index (CVI)

\begin{tabular}{|c|c|c|c|c|c|c|}
\hline \multirow{2}{*}{ Items } & \multicolumn{4}{|c|}{ Content Validity Criteria } & \multirow{2}{*}{ Mean } & \multirow{2}{*}{$\begin{array}{l}\text { Content Validity } \\
\text { Index (CVI) }\end{array}$} \\
\hline & Relevance & Clarity & Simplicity & Ambiguity & & \\
\hline \multicolumn{7}{|l|}{ Customer linkage } \\
\hline $\begin{array}{l}\text { Our enterprise interacts with customers to } \\
\text { forecast demand. }\end{array}$ & 3.33 & 3.33 & 3.00 & 2.83 & 3.13 & 0.71 \\
\hline $\begin{array}{l}\text { Our enterprise shares order status with } \\
\text { customers during order scheduling. }\end{array}$ & 2.83 & 2.17 & 2.00 & 2.50 & 2.38 & 0.63 \\
\hline $\begin{array}{l}\text { Our enterprise shares order status with } \\
\text { customers during product manufacturing. }\end{array}$ & 2.67 & 2.67 & 2.50 & 2.33 & 2.54 & 0.58 \\
\hline \multicolumn{7}{|l|}{ Internal linkage } \\
\hline $\begin{array}{l}\text { Our enterprise has access to inventory levels } \\
\text { in our supply chain. }\end{array}$ & 2.50 & 2.67 & 2.17 & 2.17 & 2.38 & 0.54 \\
\hline \multicolumn{7}{|l|}{ Operational performance } \\
\hline $\begin{array}{l}\text { Our enterprise can quickly modify products to } \\
\text { meet our major customer's requirements. }\end{array}$ & 2.67 & 2.83 & 2.83 & 2.83 & 2.79 & 0.71 \\
\hline $\begin{array}{l}\text { Our enterprise can quickly introduce new } \\
\text { products into the market. }\end{array}$ & 2.67 & 2.83 & 2.83 & 2.83 & 2.79 & 0.71 \\
\hline $\begin{array}{l}\text { The lead time for fulfilling customers' orders } \\
\text { (the time which elapses between the receipt of } \\
\text { customer's order and the delivery of the } \\
\text { goods) is short. }\end{array}$ & 3.00 & 3.00 & 1.83 & 2.50 & 2.58 & 0.63 \\
\hline
\end{tabular}

Table 4: The Improvised Version of Items

\begin{tabular}{|c|c|c|}
\hline Dimensions & No. of Items & Items \\
\hline Customer & 1. & Our enterprise shares product information with customers by online (e.g. social media, e-mail, website etc.). \\
\hline \multirow[t]{8}{*}{ Linkage } & 2. & Our enterprise shares product information with customers by phone call. \\
\hline & 3. & Our enterprise shares product information with customers by counter. \\
\hline & 4. & Our enterprise accepts customers' booking by online (e.g. social media, e-mail website etc.). \\
\hline & 5. & Our enterprise accepts customers' booking by phone call. \\
\hline & 6. & Our enterprise accepts customers' booking by counter. \\
\hline & 7. & Our enterprise has a booking system that is fast. \\
\hline & 8. & Our enterprise has a booking system that is easy to access. \\
\hline & 9. & Our enterprise shares booking status with customers during service delivery. \\
\hline Supplier & 1. & Our enterprise has strategic alliance with suppliers in our supply chain. \\
\hline \multirow[t]{6}{*}{ Linkage } & 2. & Our enterprise involves suppliers during the design stage for our new services. \\
\hline & 3. & Our enterprise involves suppliers in planning the services. \\
\hline & 4. & Our enterprise involves suppliers in inventory management. \\
\hline & 5. & Our enterprise responds to booking processing with our suppliers. \\
\hline & 6. & Our enterprise has network with suppliers that assure reliable delivery. \\
\hline & 7. & Our enterprise uses information technology to exchange information with suppliers. \\
\hline Internal & 1. & Our enterprise has a computerised database for service offerings. \\
\hline \multirow[t]{8}{*}{ Linkage } & 2. & Our enterprise has computerised database for distribution. \\
\hline & 3. & Our enterprise has a computerised database for supplier information. \\
\hline & 4. & Our enterprise has easy access to operational data in a computerised database. \\
\hline & 5. & Our enterprise has a computerised information system to link our internal departments. \\
\hline & 6. & Our enterprise can retrieve inventory status in real time. \\
\hline & 7. & Our enterprise utilizes a computerised marketing system. \\
\hline & 8. & Our enterprise utilizes a computerised service offering system. \\
\hline & 9. & Our enterprise has information system that integrates with service offering process. \\
\hline Operational & 1. & Our enterprise can respond to changes in market demand. \\
\hline \multirow[t]{2}{*}{ Performance } & 2. & Our enterprise has an on-time delivery record in our system. \\
\hline & 3. & Our enterprise provides customer service to our customers. \\
\hline Financial & 1. & Growth in sales. \\
\hline \multirow[t]{6}{*}{ Performance } & 2. & Return on sales. \\
\hline & 3. & Growth in return on sales. \\
\hline & 4. & Growth in profit. \\
\hline & 5. & Growth in market share. \\
\hline & 6. & Return on investment. \\
\hline & 7. & Growth in return on investment. \\
\hline
\end{tabular}


From the findings, several items need modification due to some issues such as double-barrel, use of jargons and incompatibility of existing items to measure the information linkage in service sector for small and medium enterprises. Not only as the items were adopted from researches done in other countries (Flynn et al., 2010; Lee et al., 2007) where the content validity adjustment is required to ensure that the instrument items will fit into Malaysia SMEs context, the adjustment is also needed due to the fact that the authors' items in the research instrument to measure the information linkage in service sector are not the same with items in research instrument for manufacturing sector as these two sectors have different characteristics, especially in term of offering physical goods versus intangible services. Thus, the need to modify the items of the research instrument justifies the importance of this paper.

The judgements and recommendations of experts from both industry and academic world are crucial as they are thoughtful on various business sectors. By documenting the content validity of the research instrument, the authors hope to contribute and path the way for future researchers, be it in content validity and/or information sharing linkage.

\section{ACKNOWLEDGEMENT}

This paper is related to the RAGS grant: RAGS/SS05(1)/1183/2014 and supported by the Universiti Malaysia Sarawak (UNIMAS).

\section{REFERENCES}

Elanna, S. Y., \& Popham, W. J. (1983). Content validity at the crossroads. American Educational Research Association, 12(8), 10-21.

Flynn, B. B., Huo, B., \& Zhao, X. (2010). The impact of supply chain integration on performance: A contingency and configuration approach. Journal of Operations Management, 28(1), 58-71.

Guion, R. M. (1977). Content validity - The source of my discontent. Applied Psychological Measurement, 1(1), 1-10.

Haynes, S. N., Richard, D. C. S., \& Kubany, E. S. (1995). Content validity in psychological assessment : A functional approach to concepts and methods. American Psychological Association, 7(3), 238247.

Ibrahim, S. E., \& Ogunyemi, O. (2012). The effect of linkages and information sharing on supply chain and export performance An empirical study of Egyptian textile manufacturers. Journal of Manufacturing Technology Management, 23(4), 441-463. doi:10.1108/17410381211230394

Lawshe, C. H. (1975). A quantitative approach to content validity. Personnel Psychology, 28(4), 563575.

Lee, C. W., Kwon, I.-W. G., \& Severance, D. (2007). Relationship between supply chain performance and degree of linkage among supplier, internal integration, and customer. Supply Chain Management: An International Journal, 6(12), 444-452. doi:10.1108/13598540710826371

Lloyd, S. (2011). Applying the nominal group technique to specify the domain of a construct. Qualitative Market Research: An International Journal, 14(1), 105-121.

Polit, D. F., \& Beck, C. T. (2006). The Content Validity Index : Are You Sure You Know What's Being Reported? Critique and Recommendations. Research in Nursing \& Health, 29(5), 489497.

Rungtusanatham, M. (1998). Let's Not Overlook Content Validity. Decision Line, (July), 10-13. 
Samiee, S., Yip, L. S. C., \& Luk, S. T. K. (2004). International marketing in Southeast Asia: Retailing trends and opportunities in China. International Marketing Review, 21(3), 247-254.

Sireci, S. G. (1998). The construct of content validity. Social Indicators Research, 45(1/3), 83-117.

Waltz, C. F. \& Bausell, R. B. (1981). Nursing research: design, statistics, and computer analysis. Philadelphia: F.A. Davis Co.

Wynd, C. A., Schmidt, B., \& Schaefer, M. A. (2003). Two Quantitative Approaches for Estimating Content Validity. Western Journal of Nursing Research, 25(5), 508-518.

Yaghmaie, F. (2003). Content validity and its estimation. Journal of Medical Education, 3(1), 25-27. 